\title{
Synthesis and Antimicrobial Screening of Quinazolone Containing Novel Heterocyclic Schiff Base and Azetidinone by Niementowski Reaction
}

\author{
A. R. DESAI, R. U. ROY and K.R.DESAI*. \\ Department of chemistry, \\ Veer Narmad South Gujarat University, \\ Surat-395007, India.
}

Received 29 November 2004; Accepted 20 February 2005

\begin{abstract}
Several 2-[2-\{(4-substitutedbenzylidene)-phenyl $\}$ vinyl]-4-oxo-3,4dihydroquinazolone-6-sulfonicacid 4(a-l) and 2-[2-\{4-(3-chloro-4-substituedphenylazetidin-2-one)-phenyl \} vinyl]-4-oxo-3,4-dihydroqui-nazolone-6-sulfonic acid 5(a-l) were synthesized by using conventional techniques and were screened for antibacterial and antifungal activity. The compounds showed good to moderate antimicrobial activity and were characterized on the basis of spectral analysis.
\end{abstract}

Key words: Niementowski reaction, Quinazolone, Schiff Base, Azetidinone, Antibacterial, Antifungal

\section{Introduction}

Quinazolone derivatives attract a widespread interest due to the diverse biological activities ${ }^{1}$ associated with them. They are pharmaceutically important as antituberculars ${ }^{2}$, antibacterial ${ }^{3}$, antiparkinsons ${ }^{4}$ and they also show blood platelet anti-aggregating activity. ${ }^{5}$ Formation of 2-alkyl-4 $(3 \mathrm{H})$ quinazolinones by condensation of anthranilic acid or substituted anthranilic acid and amides is designated as the Niementowski reaction. ${ }^{6}$

Schiff base posses diversified biological applications ${ }^{7-8}$. Azetidinone also possess a variety of pharmacological properties viz CNS depressant ${ }^{9}$, anticonvulsant ${ }^{10}$, amebicidal ${ }^{11}$, fungicidal and antibacterial $^{12}$. In a view of these observations we thought if of interest to undertake the synthesis and bioassay of compounds having quinazolone nucleus along with Schiff Base and Azetidinone moieties in a single molecular framework as possible antibacterial and antifungal agents. The present paper reports the synthesis of some new Schiff Base and Azetidinone in quinazolone by niementowski reaction.

Condensation of 5-Sulpho anthranilic acid with Acetyl Chloride in presence of pyridine gave 2-methyl-6-sulpho-4H-3, 1-benzoxazin-4-one which on reaction with ammonia gave the 2-methyl4-oxo-3,4-dihydroquinazolin-6-sulfonic acid (1). This on further reaction with 4-nitro benzaldehyde and consequent reduction gave 2-[2-(4-aminophenyl) vinyl]-4-oxo-3, 4- dihydroquinazolone-6sulfonic acid (3). Condensation with substituted aldehydes yields the title compounds 
2-[2-\{(4-substitutedbenzylidene)-phenyl $\}$ vinyl]-4-oxo-3,4-dihydroquin-azolone-6-sulfonic acid 4(a-l) which on further reaction with CAC (Chloro Acetyl Chloride) in presence of TEA (triethyl amine) in catalytic amount resulted into titled compound 2-[2-\{4-(3-chloro-4-substituedphenyl-azetidin-2-one)phenyl \} vinyl]-4-oxo-3,4-dihydroquin-azolone -6-sulfonic acid 5(a-l). The compounds are confirmed by their elemental analysis (Table-I), IR spectral analysis (Table-IIa) and ${ }^{1} \mathrm{H}$ NMR spectral analysis (Table-IIb).

\section{Experimental}

All melting points were determined in PMP-DM scientific melting point apparatus and are uncorrected. The IR spectra were recorded in $\mathrm{KBr}$ on a "Perkin-Elmer RX 1 FT-IR" Spectrophotometer (serial No. 51448) and ${ }^{1} \mathrm{H}$ NMR spectra was recorded in $\mathrm{CDCl}_{3}$ as well as mixture of $\mathrm{CDCl}_{3}+\mathrm{DMSO}$ with TMS as an internal Standard on $300 \mathrm{MHz}$ "Bruker $\mathrm{A} / \mathrm{C} 300 \mathrm{~F}$ Spectrophotometer".

Preparation of 2-methyl-4-oxo-3,4-dihydroquinazolin-6-sulfonic acid (1):

In a $250 \mathrm{~mL}$ Beaker 5-Sulpho anthranilic acid $(2.17 \mathrm{~g}, 0.01 \mathrm{M})$ in presence of pyridine $(0.79 \mathrm{~g}, 0.01 \mathrm{M})$ were taken. Acetyl chloride $(0.78 \mathrm{~g}, 0.01 \mathrm{M})$ at $0^{\circ}-5^{\circ} \mathrm{C}$ was added drop wise to afford cyclization to give 2-methyl-6-sulpho-4H-3, 1-benzoxazin-4-one. The resulting compound on treatment with ammonia gave 2-methyl-4-oxo-3,4-dihydroquinazolin-6-sulfonic acid (1). The resulting solid was filetered, washed, dried and recrystallised from glacial acetic acid. The \% yield was $85 \%$, m.p $225^{\circ} \mathrm{C}$.

Preparation of 2-[2-(4-nitrophenyl) vinyl]-4-oxo-3,4-dihydroquinaz-oline-6-sulphonic acid (2):

In a $250 \mathrm{~mL}$ R.B.F a mixture of 2-methyl-4-oxo-3,4-dihydroquinazolin-6-sulfonic acid (1) (3.63g, $0.01 \mathrm{M})$ and $4-\mathrm{Nitro}$ benzaldehyde $(1.51 \mathrm{~g}, 0.01 \mathrm{M})$ in acetic anhydride was taken. The reaction mixture was refluxed for 6-8 hrs and cooled to room temperature. The excess of solvent was then distilled off and then the contents were poured on to crushed ice. The resulting solid was filtered, washed, dried and recrystallised from glacial acetic acid. The \% yield was $75 \%$, m. p $210^{\circ} \mathrm{C}$.

Preparation of 2-[2-(4-aminophenyl) vinyl]-4-oxo-3,4-dihydroquinaz-oline-6-sulphonic acid (3):

In a $250 \mathrm{~mL}$ R.B.F 2-[2-(4-nitrophenyl) vinyl]-4-oxo-3,4-dihydroquinazoline-6-sulphonic acid (2) $(7.46 \mathrm{~g}, 0.02 \mathrm{M})$ was taken. The solution of sodium sulphide $(0.06 \mathrm{M})$ in water $(75 \mathrm{~mL})$ was added to it. The reaction mixture was refluxed for 2-3 hrs. A deep reddish brown solution was obtained. After cooling, it was diluted with water. The conc. $\mathrm{HCl}$ was added to the solution and was boiled gently till the removal of $\mathrm{H}_{2} \mathrm{~S}$ was ceased completely. Neutralization with sodium carbonate precipitate 2-[2-(4aminophenyl) vinyl]-4-oxo-3,4-dihydroquinazoline-6-sulphonic acid (3) as free amino compound as yellow product. The product was filtered, washed, dried and recrystallised from ethanol. The \% yield is $60 \%$, m.p $281^{\circ} \mathrm{C}$.

Preparation of 2-[2-\{(4-substitutedbenzylidene)-phenyl\} vinyl]-4-oxo-3, 4-dihydroquinazolone-6sulfonic acid 4(a-l):-

In a $250 \mathrm{~mL}$ R.B.F 2-[2-(4-aminophenyl) vinyl]-4-oxo-3,4-dihydroquinazoline-6-sulphonic acid (3) $(3.43 \mathrm{~g}, 0.01 \mathrm{M})$ and substituted benzaldehyde $(0.01 \mathrm{M})$ were taken in methanol $(30 \mathrm{~mL})$ as a solvent. The reaction mixture was refluxed with the help of reflux condenser attached with a dean-stark apparatus, till azeotropic removal of water. The reaction was carried out in presence of an acid catalyst. The resulting mixture was cooled and poured into crushed ice. The solid obtained was filtered, washed, dried and recrystallised by methanol. The \% yield was 80\%. IR: $v$ max $1630(\mathrm{C}=\mathrm{N}), 1713(\mathrm{C}=\mathrm{O}), 1410$ $(\mathrm{CH}=\mathrm{CH}), 1670(>\mathrm{N}=\mathrm{CH}-) ;{ }^{1} \mathrm{H}$ NMR $(\mathrm{CDCl} 3+\mathrm{DMSO}$; TMS as internal standard): $\delta 7.1-8.1(\mathrm{~m}, 3 \mathrm{H}$, $\mathrm{H}-5$, and H-7-8), $\delta 9.1$ (broad, s, $1 \mathrm{H}, \mathrm{NH}), \delta 2.5(\mathrm{~d}, 1 \mathrm{H}, \mathrm{CH}=\mathrm{CH}), \delta 2.7(\mathrm{~d}, 1 \mathrm{H}, \mathrm{CH}=\mathrm{CH})$. 
Table-1. Elemental Analysis of compound 4 (a-l) \& 5(a-l)

\begin{tabular}{|c|c|c|c|c|c|c|c|c|c|}
\hline \multirow{2}{*}{$\begin{array}{c}\text { Comp } \\
\text { No. }\end{array}$} & \multirow{2}{*}{$\begin{array}{l}\text { Formula } \\
\left(M . P /{ }^{\circ} \mathrm{C}\right)\end{array}$} & \multicolumn{3}{|c|}{ Anal. Calcd. (found) / \% } & \multirow{2}{*}{$\begin{array}{c}\text { Comp } \\
\text { No. }\end{array}$} & \multirow{2}{*}{$\begin{array}{l}\text { Formula } \\
\left(M . P /{ }^{\circ} C\right)\end{array}$} & \multicolumn{3}{|c|}{ Anal. Calcd. (found) / \% } \\
\hline & & $\mathbf{C}$ & $\mathbf{H}$ & $\mathbf{N}$ & & & $\mathbf{C}$ & $\mathbf{H}$ & $\mathbf{N}$ \\
\hline $4 \mathbf{a}$ & $\begin{array}{c}\mathrm{C}_{25} \mathrm{H}_{22} \mathrm{~N}_{4} \mathrm{O}_{4} \mathrm{~S} \\
\text { (84) }\end{array}$ & $\begin{array}{l}63.28 \\
(63.32\end{array}$ & $\begin{array}{l}4.67 \\
4.64\end{array}$ & $\begin{array}{c}11.81 \\
11.83)\end{array}$ & $5 \mathbf{a}$ & $\begin{array}{c}\mathrm{C}_{27} \mathrm{H}_{23} \mathrm{ClN}_{4} \mathrm{O}_{5} \mathrm{~S} \\
(182)\end{array}$ & $\begin{array}{l}58.85 \\
(58.83\end{array}$ & $\begin{array}{l}4.21 \\
4.25\end{array}$ & $\begin{array}{r}10.17 \\
10.21)\end{array}$ \\
\hline $4 b$ & $\begin{array}{c}\mathrm{C}_{23} \mathrm{H}_{16} \mathrm{ClN}_{3} \mathrm{O}_{4} \mathrm{~S} \\
(102)\end{array}$ & $\begin{array}{r}59.29 \\
(59.24\end{array}$ & $\begin{array}{l}3.46 \\
3.42\end{array}$ & $\begin{array}{l}9.02 \\
9.05)\end{array}$ & $5 \mathbf{b}$ & $\begin{array}{c}\mathrm{C}_{25} \mathrm{H}_{17} \mathrm{Cl}_{2} \mathrm{~N}_{3} \mathrm{O}_{5} \mathrm{~S} \\
(210)\end{array}$ & $\begin{array}{l}55.36 \\
(55.40\end{array}$ & $\begin{array}{l}3.16 \\
3.18\end{array}$ & $\begin{array}{c}7.75 \\
7.81)\end{array}$ \\
\hline $4 c$ & $\begin{array}{c}\mathrm{C}_{23} \mathrm{H}_{16} \mathrm{ClN}_{3} \mathrm{O}_{4} \mathrm{~S} \\
(210)\end{array}$ & $\begin{array}{l}59.35 \\
(59.38\end{array}$ & $\begin{array}{l}3.52 \\
3.49\end{array}$ & $\begin{array}{l}9.28 \\
9.25)\end{array}$ & $5 c$ & $\begin{array}{c}\mathrm{C}_{25} \mathrm{H}_{17} \mathrm{Cl}_{2} \mathrm{~N}_{3} \mathrm{O}_{5} \mathrm{~S} \\
(225)\end{array}$ & $\begin{array}{l}55.52 \\
(55.49\end{array}$ & $\begin{array}{l}3.25 \\
3.21\end{array}$ & $\begin{array}{c}7.91 \\
7.94)\end{array}$ \\
\hline $4 d$ & $\begin{array}{c}\mathrm{C}_{23} \mathrm{H}_{17} \mathrm{~N}_{3} \mathrm{O}_{5} \mathrm{~S} \\
(192)\end{array}$ & $\begin{array}{r}61.74 \\
(61.72\end{array}$ & $\begin{array}{l}3.83 \\
3.85\end{array}$ & $\begin{array}{l}9.39 \\
9.42)\end{array}$ & $5 d$ & $\begin{array}{c}\mathrm{C}_{25} \mathrm{H}_{18} \mathrm{ClN}_{3} \mathrm{O}_{6} \mathrm{~S} \\
(190)\end{array}$ & $\begin{array}{l}57.31 \\
(57.34\end{array}$ & $\begin{array}{l}3.46 \\
3.42\end{array}$ & $\begin{array}{l}8.02 \\
8.05)\end{array}$ \\
\hline $4 e$ & $\begin{array}{c}\mathrm{C}_{23} \mathrm{H}_{17} \mathrm{~N}_{3} \mathrm{O}_{5} \mathrm{~S} \\
(225)\end{array}$ & $\begin{array}{l}61.89 \\
(61.92\end{array}$ & $\begin{array}{l}3.73 \\
3.78\end{array}$ & $\begin{array}{l}9.52 \\
9.50)\end{array}$ & $5 e$ & $\begin{array}{c}\mathrm{C}_{25} \mathrm{H}_{18} \mathrm{ClN}_{3} \mathrm{O}_{6} \mathrm{~S} \\
(125)\end{array}$ & $\begin{array}{l}57.82 \\
(57.79\end{array}$ & $\begin{array}{l}3.05 \\
3.07\end{array}$ & $\begin{array}{l}8.25 \\
8.27)\end{array}$ \\
\hline $4 f$ & $\begin{array}{c}\mathrm{C}_{23} \mathrm{H}_{16} \mathrm{~N}_{4} \mathrm{O}_{6} \mathrm{~S} \\
(280)\end{array}$ & $\begin{array}{c}57.95 \\
(57.98\end{array}$ & $\begin{array}{l}3.38 \\
3.42\end{array}$ & $\begin{array}{l}11.76 \\
11.78)\end{array}$ & $5 f$ & $\begin{array}{c}\mathrm{C}_{25} \mathrm{H}_{17} \mathrm{ClN}_{4} \mathrm{O}_{7} \mathrm{~S} \\
(252)\end{array}$ & $\begin{array}{r}54.30 \\
(54.27\end{array}$ & $\begin{array}{l}3.10 \\
3.12\end{array}$ & $\begin{array}{r}10.13 \\
10.11)\end{array}$ \\
\hline $4 \mathrm{~g}$ & $\begin{array}{c}\mathrm{C}_{23} \mathrm{H}_{16} \mathrm{~N}_{4} \mathrm{O}_{6} \mathrm{~S} \\
(>300)\end{array}$ & $\begin{array}{l}57.75 \\
(57.72\end{array}$ & $\begin{array}{l}3.45 \\
3.48\end{array}$ & $\begin{array}{r}11.55 \\
11.52)\end{array}$ & $5 \mathrm{~g}$ & $\begin{array}{c}\mathrm{C}_{25} \mathrm{H}_{17} \mathrm{ClN}_{4} \mathrm{O}_{7} \mathrm{~S} \\
(272)\end{array}$ & $\begin{array}{l}54.62 \\
(54.58\end{array}$ & $\begin{array}{l}3.85 \\
3.82\end{array}$ & $\begin{array}{l}10.72 \\
10.75)\end{array}$ \\
\hline $4 h$ & $\begin{array}{c}\mathrm{C}_{24} \mathrm{H}_{19} \mathrm{~N}_{3} \mathrm{O}_{6} \mathrm{~S} \\
(255)\end{array}$ & $\begin{array}{l}60.37 \\
(60.32\end{array}$ & $\begin{array}{l}4.01 \\
4.05\end{array}$ & $\begin{array}{r}8.80 \\
8.82)\end{array}$ & $5 \mathbf{h}$ & $\begin{array}{c}\mathrm{C}_{25} \mathrm{H}_{17} \mathrm{BrClN}_{3} \mathrm{O}_{5} \mathrm{~S} \\
(210)\end{array}$ & $\begin{array}{l}51.17 \\
(51.21\end{array}$ & $\begin{array}{l}2.92 \\
2.89\end{array}$ & $\begin{array}{l}7.16 \\
7.12)\end{array}$ \\
\hline $4 \mathbf{i}$ & $\begin{array}{c}\mathrm{C}_{23} \mathrm{H}_{16} \mathrm{BrN}_{3} \mathrm{O}_{4} \mathrm{~S} \\
(270)\end{array}$ & $\begin{array}{c}54.13 \\
(54.15\end{array}$ & $\begin{array}{l}3.16 \\
3.12\end{array}$ & $\begin{array}{l}8.23 \\
8.27)\end{array}$ & $5 \mathbf{i}$ & $\begin{array}{c}\mathrm{C}_{26} \mathrm{H}_{20} \mathrm{ClN}_{3} \mathrm{O}_{7} \mathrm{~S} \\
(182)\end{array}$ & $\begin{array}{l}56.37 \\
(56.41\end{array}$ & $\begin{array}{l}3.64 \\
3.62\end{array}$ & $\begin{array}{l}7.59 \\
7.63)\end{array}$ \\
\hline $\mathbf{4 j}$ & $\begin{array}{c}\mathrm{C}_{24} \mathrm{H}_{19} \mathrm{~N}_{3} \mathrm{O}_{5} \mathrm{~S} \\
(200)\end{array}$ & $\begin{array}{l}62.46 \\
(62.49\end{array}$ & $\begin{array}{l}4.15 \\
4.12\end{array}$ & $\begin{array}{l}9.11 \\
9.12)\end{array}$ & $5 \mathbf{j}$ & $\begin{array}{c}\mathrm{C}_{26} \mathrm{H}_{20} \mathrm{ClN}_{3} \mathrm{O}_{6} \mathrm{~S} \\
(290)\end{array}$ & $\begin{array}{l}58.05 \\
(58.09\end{array}$ & $\begin{array}{l}3.75 \\
3.78\end{array}$ & $\begin{array}{l}7.81 \\
7.84)\end{array}$ \\
\hline $4 k$ & $\begin{array}{c}\mathrm{C}_{23} \mathrm{H}_{15} \mathrm{Cl}_{2} \mathrm{~N}_{3} \mathrm{O}_{4} \mathrm{~S} \\
(210)\end{array}$ & $\begin{array}{l}55.21 \\
(55.25\end{array}$ & $\begin{array}{l}3.02 \\
3.05\end{array}$ & $\begin{array}{l}8.40 \\
8.44)\end{array}$ & $5 \mathbf{k}$ & $\begin{array}{c}\mathrm{C}_{25} \mathrm{H}_{16} \mathrm{Cl}_{3} \mathrm{~N}_{3} \mathrm{O}_{5} \mathrm{~S} \\
(152)\end{array}$ & $\begin{array}{l}52.05 \\
(52.07\end{array}$ & $\begin{array}{l}2.80 \\
2.79\end{array}$ & $\begin{array}{l}7.28 \\
7.30)\end{array}$ \\
\hline 41 & $\begin{array}{c}\mathrm{C}_{29} \mathrm{H}_{21} \mathrm{~N}_{3} \mathrm{O}_{5} \mathrm{~S} \\
(240)\end{array}$ & $\begin{array}{r}66.53 \\
(66.55 \\
\end{array}$ & $\begin{array}{l}4.04 \\
4.01 \\
\end{array}$ & $\begin{array}{r}8.03 \\
8.07) \\
\end{array}$ & 51 & $\begin{array}{c}\mathrm{C}_{31} \mathrm{H}_{22} \mathrm{ClN}_{3} \mathrm{O}_{6} \mathrm{~S} \\
(221)\end{array}$ & $\begin{array}{r}62.05 \\
(62.07 \\
\end{array}$ & $\begin{array}{l}3.70 \\
3.72 \\
\end{array}$ & $\begin{array}{l}7.00 \\
7.02) \\
\end{array}$ \\
\hline & & $\begin{array}{c}\text { Comp. No } \\
\mathbf{4 a - 5 a} \\
\mathbf{4 b - 5 b} \\
\mathbf{4 c - 5 c} \\
\mathbf{4 d - 5 d} \\
\mathbf{4 e - 5 e} \\
\mathbf{4 f - 5 f} \\
\end{array}$ & $\begin{array}{r}\mathrm{H} \\
4-\mathrm{N}( \\
4- \\
2- \\
4-\mathrm{C} \\
2-( \\
4-\mathrm{N}\end{array}$ & & $\begin{array}{c}\text { Comp. No } \\
4 \mathbf{g}-5 \mathrm{~g} \\
\mathbf{4 h - 5 h} \\
4 \mathbf{i}-5 \mathbf{i} \\
4 \mathbf{j}-5 \mathbf{j} \\
4 \mathbf{k}-5 \mathbf{k} \\
4 \mathbf{l}-5 \mathbf{l} \\
\end{array}$ & $\begin{array}{r}\mathrm{R} \\
2-\mathrm{NO}_{2} \\
2-\mathrm{Br} \\
3-\mathrm{OH}, 4-\mathrm{O} \\
3-\mathrm{OCH}_{3} \\
2: 4-\mathrm{Cl}_{2} \\
3-\mathrm{OC}_{6} \mathrm{H} \\
\end{array}$ & & & \\
\hline
\end{tabular}


Table-IIa . IR Spectral Analysis of compound 4(a-l) and 5(a-l)

\begin{tabular}{|c|c|c|c|}
\hline Comp & IR (KBr) & Comp & IR (KBr) \\
\hline No. & $v_{\max } / \mathrm{cm}^{-1}$ & No. & $v_{\max } / \mathrm{cm}^{-1}$ \\
\hline $\mathbf{4 a}$ & $\begin{array}{l}1595(\mathrm{C}=\mathrm{N}), 1710(\mathrm{C}=\mathrm{O}), 1410(-\mathrm{CH}=\mathrm{CH}-) \\
1670(>\mathrm{N}=\mathrm{CH}-)\end{array}$ & $5 \mathbf{a}$ & $\begin{array}{l}1630(\mathrm{C}=\mathrm{N}), 1710(\mathrm{C}=\mathrm{O}), 1420(-\mathrm{CH}=\mathrm{CH}-) \\
1730(-\mathrm{C}=\mathrm{O}), 785(\mathrm{C}-\mathrm{Cl})\end{array}$ \\
\hline $4 b$ & $\begin{array}{l}1580(\mathrm{C}=\mathrm{N}), 1705(\mathrm{C}=\mathrm{O}), 1412(-\mathrm{CH}=\mathrm{CH}-) \\
1675(>\mathrm{N}=\mathrm{CH}-), 775(\mathrm{C}-\mathrm{Cl})\end{array}$ & $5 b$ & $\begin{array}{l}1580(\mathrm{C}=\mathrm{N}), 1705(\mathrm{C}=\mathrm{O}), 1412(-\mathrm{CH}=\mathrm{CH}-) \\
1725(\mathrm{C}=\mathrm{O}), 775(\mathrm{C}-\mathrm{Cl})\end{array}$ \\
\hline $4 c$ & $\begin{array}{l}1605(\mathrm{C}=\mathrm{N}), 1700(\mathrm{C}=\mathrm{O}), 1415(-\mathrm{CH}=\mathrm{CH}-) \\
1645(>\mathrm{N}=\mathrm{CH}-), 785(\mathrm{C}-\mathrm{Cl})\end{array}$ & $5 c$ & $\begin{array}{l}1605(\mathrm{C}=\mathrm{N}), 1700(\mathrm{C}=\mathrm{O}), 1415(-\mathrm{CH}=\mathrm{CH}-) \\
1728(\mathrm{C}=\mathrm{O}), 780(\mathrm{C}-\mathrm{Cl})\end{array}$ \\
\hline 4d & $\begin{array}{l}1590(\mathrm{C}=\mathrm{N}), 1715(\mathrm{C}=\mathrm{O}), 1411(-\mathrm{CH}=\mathrm{CH}-) \\
1652(>\mathrm{N}=\mathrm{CH}-), 3310(\mathrm{Ar}-\mathrm{OH})\end{array}$ & $5 d$ & $\begin{array}{l}1590(\mathrm{C}=\mathrm{N}), 1715(\mathrm{C}=\mathrm{O}), 1411(-\mathrm{CH}=\mathrm{CH}-) \\
1715(\mathrm{C}=\mathrm{O}), 765(\mathrm{C}-\mathrm{Cl}), 3310(\mathrm{Ar}-\mathrm{OH})\end{array}$ \\
\hline $4 e$ & $\begin{array}{l}1595(\mathrm{C}=\mathrm{N}), 1708(\mathrm{C}=\mathrm{O}), 1410(-\mathrm{CH}=\mathrm{CH}-) \\
1670(>\mathrm{N}=\mathrm{CH}-), 3380(\text { Ar-OH })\end{array}$ & $5 e$ & $\begin{array}{l}1595(\mathrm{C}=\mathrm{N}), 1708(\mathrm{C}=\mathrm{O}), 1410(-\mathrm{CH}=\mathrm{CH}-) \\
1720(\mathrm{C}=\mathrm{O}), 758(\mathrm{C}-\mathrm{Cl}), 3380(\mathrm{Ar}-\mathrm{OH})\end{array}$ \\
\hline $4 f$ & $\begin{array}{l}1610(\mathrm{C}=\mathrm{N}), 1720(\mathrm{C}=\mathrm{O}), 1418(-\mathrm{CH}=\mathrm{CH}-) \\
1672(>\mathrm{N}=\mathrm{CH}-), 1370,1542\left(\mathrm{Ar}-\mathrm{NO}_{2}\right)\end{array}$ & $5 f$ & $\begin{array}{l}1610(\mathrm{C}=\mathrm{N}), 1720(\mathrm{C}=\mathrm{O}), 1418(-\mathrm{CH}=\mathrm{CH}-) \\
1725(\mathrm{C}=\mathrm{O}), 785(\mathrm{C}-\mathrm{Cl}), 1370,1542\left(\mathrm{Ar}-\mathrm{NO}_{2}\right)\end{array}$ \\
\hline $4 g$ & $\begin{array}{l}1600(\mathrm{C}=\mathrm{N}), 1695(\mathrm{C}=\mathrm{O}), 1405(-\mathrm{CH}=\mathrm{CH}-) \\
1665(>\mathrm{N}=\mathrm{CH}-), 1365,1550\left(\mathrm{Ar}-\mathrm{NO}_{2}\right)\end{array}$ & $5 g$ & $\begin{array}{l}1600(\mathrm{C}=\mathrm{N}), 1695(\mathrm{C}=\mathrm{O}), 1405(-\mathrm{CH}=\mathrm{CH}-) \\
1720(\mathrm{C}=\mathrm{O}), 790(\mathrm{C}-\mathrm{Cl}), 1365,1550\left(\mathrm{Ar}-\mathrm{NO}_{2}\right)\end{array}$ \\
\hline $4 h$ & $\begin{array}{l}1595(\mathrm{C}=\mathrm{N}), 1705(\mathrm{C}=\mathrm{O}), 1418(-\mathrm{CH}=\mathrm{CH}-) \\
1675(>\mathrm{N}=\mathrm{CH}-), 545(\mathrm{C}-\mathrm{Br})\end{array}$ & $5 h$ & $\begin{array}{l}1595(\mathrm{C}=\mathrm{N}), 1705(\mathrm{C}=\mathrm{O}), 1418(-\mathrm{CH}=\mathrm{CH}-) \\
1710(\mathrm{C}=\mathrm{O}), 765(\mathrm{C}-\mathrm{Cl}), 545(\mathrm{C}-\mathrm{Br})\end{array}$ \\
\hline $4 i$ & $\begin{array}{l}1590(\mathrm{C}=\mathrm{N}), 1715(\mathrm{C}=\mathrm{O}), 1410(-\mathrm{CH}=\mathrm{CH}-) \\
1670(>\mathrm{N}=\mathrm{CH}-), 3310(\mathrm{Ar}-\mathrm{OH}), 2825\left(\mathrm{C}-\mathrm{OCH}_{3}\right)\end{array}$ & $5 i$ & $\begin{array}{l}1590(\mathrm{C}=\mathrm{N}), 1715(\mathrm{C}=\mathrm{O}), 1410(-\mathrm{CH}=\mathrm{CH}-) \\
1725(\mathrm{C}=\mathrm{O}), 795(\mathrm{C}-\mathrm{Cl}), 3310(\mathrm{Ar}-\mathrm{OH}), 2825\left(\mathrm{C}-\mathrm{OCH}_{3}\right)\end{array}$ \\
\hline $4 j$ & $\begin{array}{l}1595(\mathrm{C}=\mathrm{N}), 1710(\mathrm{C}=\mathrm{O}), 1410(-\mathrm{CH}=\mathrm{CH}-) \\
1670(>\mathrm{N}=\mathrm{CH}-), 2825\left(\mathrm{C}-\mathrm{OCH}_{3}\right)\end{array}$ & $5 \mathbf{j}$ & $\begin{array}{l}1595(\mathrm{C}=\mathrm{N}), 1710(\mathrm{C}=\mathrm{O}), 1410(-\mathrm{CH}=\mathrm{CH}-) \\
1732(\mathrm{C}=\mathrm{O}), 752(\mathrm{C}-\mathrm{Cl}), 2825\left(\mathrm{C}-\mathrm{OCH}_{3}\right)\end{array}$ \\
\hline $4 k$ & $\begin{array}{l}1595(\mathrm{C}=\mathrm{N}), 1710(\mathrm{C}=\mathrm{O}), 1410(-\mathrm{CH}=\mathrm{CH}-) \\
1670(>\mathrm{N}=\mathrm{CH}-), 780(\mathrm{C}-\mathrm{Cl})\end{array}$ & $5 \mathbf{k}$ & $\begin{array}{l}1595(\mathrm{C}=\mathrm{N}), 1710(\mathrm{C}=\mathrm{O}), 1410(-\mathrm{CH}=\mathrm{CH}-) \\
1725(\mathrm{C}=\mathrm{O}), 780(\mathrm{C}-\mathrm{Cl}), 780(\mathrm{C}-\mathrm{Cl})\end{array}$ \\
\hline 41 & $\begin{array}{l}1595(\mathrm{C}=\mathrm{N}), 1710(\mathrm{C}=\mathrm{O}), 1410(-\mathrm{CH}=\mathrm{CH}-) \\
1670(>\mathrm{N}=\mathrm{CH}-), 1032,1245(\mathrm{Ar}-\mathrm{O}-\mathrm{Ar})\end{array}$ & 51 & $\begin{array}{l}1595(\mathrm{C}=\mathrm{N}), 1710(\mathrm{C}=\mathrm{O}), 1410(-\mathrm{CH}=\mathrm{CH}-) \\
1725(\mathrm{C}=\mathrm{O}), 755(\mathrm{C}-\mathrm{Cl}), 1032,1245(\mathrm{Ar}-\mathrm{O}-\mathrm{Ar})\end{array}$ \\
\hline
\end{tabular}


Table-IIb. ${ }^{1}$ H NMR Spectral Analysis of compounds 4(a-l)

\begin{tabular}{|c|c|}
\hline No & ${ }^{1} H$ NMR ${ }^{(a)} \delta / p p m$ \\
\hline 4a & $\begin{array}{l}\text { 7.1-8.1 (m, 11H, 2Ph, H-5, and H-7-8), } 9.2(\text { broad, s, } 1 \mathrm{H}, \mathrm{NH}), 2.4(\mathrm{~d}, 1 \mathrm{H}, \mathrm{CH}=\mathrm{CH}), 2.8(\mathrm{~d}, 1 \mathrm{H} \text {, } \\
\mathrm{CH}=\mathrm{CH}), 2.2\left(\mathrm{~s}, 1 \mathrm{H},{\left.\mathrm{N}-\mathrm{CH}_{3}\right)}^{-1}\right.\end{array}$ \\
\hline 4b & $\begin{array}{l}\text { 7.2-8.0 (m, 11H, } 2 \mathrm{Ph}, \mathrm{H}-5 \text {, and } \mathrm{H}-7-8), 9.1(\text { broad, s, } 1 \mathrm{H}, \mathrm{NH}), 2.5(\mathrm{~d}, 1 \mathrm{H}, \mathrm{CH}=\mathrm{CH}), 2.9(\mathrm{~d}, 1 \mathrm{H} \text {, } \\
\mathrm{CH}=\mathrm{CH})\end{array}$ \\
\hline $4 c$ & $\begin{array}{l}7.0-8.2(\mathrm{~m}, 11 \mathrm{H}, 2 \mathrm{Ph}, \mathrm{H}-5 \text {, and } \mathrm{H}-7-8), 9.2(\text { broad, s, } 1 \mathrm{H}, \mathrm{NH}), 2.2(\mathrm{~d}, 1 \mathrm{H}, \mathrm{CH}=\mathrm{CH}), 2.7(\mathrm{~d}, 1 \mathrm{H} \text {, } \\
\mathrm{CH}=\mathrm{CH})\end{array}$ \\
\hline 4d & $\begin{array}{l}7.1-8.3(\mathrm{~m}, 11 \mathrm{H}, 2 \mathrm{Ph}, \mathrm{H}-5 \text {, and } \mathrm{H}-7-8), 9.1(\text { broad, s, } 1 \mathrm{H}, \mathrm{NH}), 2.3(\mathrm{~d}, 1 \mathrm{H}, \mathrm{CH}=\mathrm{CH}), 2.6(\mathrm{~d}, 1 \mathrm{H} \text {, } \\
\mathrm{CH}=\mathrm{CH}), 10.1(\mathrm{~s}, 1 \mathrm{H}, \mathrm{Ar}-\mathrm{OH})\end{array}$ \\
\hline $4 e$ & $\begin{array}{l}\text { 7.2-8.2 (m, 11H, 2Ph, H-5, and H-7-8), } 9.1 \text { (broad, s, 1H, NH), } 2.4(\mathrm{~d}, 1 \mathrm{H}, \mathrm{CH}=\mathrm{CH}), 2.7(\mathrm{~d}, 1 \mathrm{H} \text {, } \\
\mathrm{CH}=\mathrm{CH}), 10.2(\mathrm{~s}, 1 \mathrm{H}, \mathrm{Ar}-\mathrm{OH})\end{array}$ \\
\hline $4 \mathbf{f}$ & $\begin{array}{l}7.0-8.2(\mathrm{~m}, 11 \mathrm{H}, 2 \mathrm{Ph}, \mathrm{H}-5 \text {, and } \mathrm{H}-7-8), 9.2(\text { broad, s, } 1 \mathrm{H}, \mathrm{NH}), 2.5(\mathrm{~d}, 1 \mathrm{H}, \mathrm{CH}=\mathrm{CH}), 2.8(\mathrm{~d}, 1 \mathrm{H} \text {, } \\
\mathrm{CH}=\mathrm{CH})\end{array}$ \\
\hline $4 \mathrm{~g}$ & $\begin{array}{l}\text { 7.0-8.1 (m, } 11 \mathrm{H}, 2 \mathrm{Ph}, \mathrm{H}-5 \text {, and } \mathrm{H}-7-8), 9.1 \text { (broad, s, } 1 \mathrm{H}, \mathrm{NH}), 2.4(\mathrm{~d}, 1 \mathrm{H}, \mathrm{CH}=\mathrm{CH}), 2.7(\mathrm{~d}, 1 \mathrm{H} \text {, } \\
\mathrm{CH}=\mathrm{CH})\end{array}$ \\
\hline $4 h$ & $\begin{array}{l}7.2-8.3(\mathrm{~m}, 11 \mathrm{H}, 2 \mathrm{Ph}, \mathrm{H}-5 \text {, and } \mathrm{H}-7-8), 9.1(\text { broad, } \mathrm{s}, 1 \mathrm{H}, \mathrm{NH}), 2.3(\mathrm{~d}, 1 \mathrm{H}, \mathrm{CH}=\mathrm{CH}), 2.8(\mathrm{~d}, 1 \mathrm{H} \text {, } \\
\mathrm{CH}=\mathrm{CH})\end{array}$ \\
\hline $4 i$ & $\begin{array}{l}\text { 7.1-8.4 (m, 11H, 2Ph, H-5, and H-7-8), } 9.2(\text { broad, s, } 1 \mathrm{H}, \mathrm{NH}), 2.4(\mathrm{~d}, 1 \mathrm{H}, \mathrm{CH}=\mathrm{CH}), 2.9(\mathrm{~d}, 1 \mathrm{H} \text {, } \\
\mathrm{CH}=\mathrm{CH}), 3.8\left(\mathrm{~s}, 1 \mathrm{H}, \mathrm{Ar}-\mathrm{OCH}_{3}\right)\end{array}$ \\
\hline $4 \mathbf{j}$ & $\begin{array}{l}\text { 7.0-8.3 (m, 11H, 2Ph, H-5, and H-7-8), } 9.1(\text { broad, s, } 1 \mathrm{H}, \mathrm{NH}), 2.3(\mathrm{~d}, 1 \mathrm{H}, \mathrm{CH}=\mathrm{CH}), 2.8(\mathrm{~d}, 1 \mathrm{H} \text {, } \\
\mathrm{CH}=\mathrm{CH}), 3.7\left(\mathrm{~s}, 1 \mathrm{H}, \mathrm{Ar}-\mathrm{OCH}_{3}\right)\end{array}$ \\
\hline $4 \mathbf{k}$ & $\begin{array}{l}\text { 7.2-8.2 (m, } 11 \mathrm{H}, 2 \mathrm{Ph}, \mathrm{H}-5 \text {, and } \mathrm{H}-7-8), 9.2(\text { broad, } \mathrm{s}, 1 \mathrm{H}, \mathrm{NH}), 2.4(\mathrm{~d}, 1 \mathrm{H}, \mathrm{CH}=\mathrm{CH}), 2.6(\mathrm{~d}, 1 \mathrm{H} \text {, } \\
\mathrm{CH}=\mathrm{CH})\end{array}$ \\
\hline 41 & $\begin{array}{l}7.0-8.2(\mathrm{~m}, 16 \mathrm{H}, 3 \mathrm{Ph}, \mathrm{H}-5 \text {, and } \mathrm{H}-7-8), 9.1(\text { broad, s, } 1 \mathrm{H}, \mathrm{NH}), 2.5(\mathrm{~d}, 1 \mathrm{H}, \mathrm{CH}=\mathrm{CH}), 2.8(\mathrm{~d}, 1 \mathrm{H} \text {, } \\
\mathrm{CH}=\mathrm{CH})\end{array}$ \\
\hline \multicolumn{2}{|r|}{ Table-IIb. ${ }^{1}$ H NMR Spectral Analysis of compounds $\mathbf{5}(\mathbf{a}-\mathbf{l})$} \\
\hline No & ${ }^{1} \mathbf{H ~ N M R}^{(\mathrm{a})} \delta / \mathbf{p p m}$ \\
\hline $5 \mathbf{a}$ & $\begin{array}{l}\text { 7.1-8.1 (m, 11H, 2Ph, H-5, and H-7-8), 9.2 (broad, s, } 1 \mathrm{H}, \mathrm{NH}), 2.4(\mathrm{~d}, 1 \mathrm{H}, \mathrm{CH}=\mathrm{CH}), 2.8(\mathrm{~d}, \\
1 \mathrm{H}, \mathrm{CH}=\mathrm{CH}), 2.2\left(\mathrm{~s}, 1 \mathrm{H}, \mathrm{N}-\mathrm{CH}_{3}\right), 2.5(1 \mathrm{H}, \mathrm{d}, \mathrm{N}-\mathrm{CH}), 4.5(1 \mathrm{H}, \mathrm{d}, \mathrm{CH}-\mathrm{Cl})\end{array}$ \\
\hline $5 \mathbf{b}$ & $\begin{array}{l}\text { 7.2-8.0 (m, 11H, 2Ph, H-5, and H-7-8), } 9.1(\text { broad, } \mathrm{s}, 1 \mathrm{H}, \mathrm{NH}), 2.5(\mathrm{~d}, 1 \mathrm{H}, \mathrm{CH}=\mathrm{CH}), 2.9(\mathrm{~d}, \\
1 \mathrm{H}, \mathrm{CH}=\mathrm{CH}), 2.4(1 \mathrm{H}, \mathrm{d}, \mathrm{N}-\mathrm{CH}), 4.4(1 \mathrm{H}, \mathrm{d}, \mathrm{CH}-\mathrm{Cl})\end{array}$ \\
\hline $5 \mathbf{c}$ & $\begin{array}{l}\text { 7.0-8.2 (m, 11H, 2Ph, H-5, and H-7-8), } 9.2(\text { broad, } \mathrm{s}, 1 \mathrm{H}, \mathrm{NH}), 2.2(\mathrm{~d}, 1 \mathrm{H}, \mathrm{CH}=\mathrm{CH}), 2.7(\mathrm{~d}, \\
1 \mathrm{H}, \mathrm{CH}=\mathrm{CH}), 2.6(1 \mathrm{H}, \mathrm{d}, \mathrm{N}-\mathrm{CH}), 4.5(1 \mathrm{H}, \mathrm{d}, \mathrm{CH}-\mathrm{Cl})\end{array}$ \\
\hline $5 d$ & $\begin{array}{l}\text { 7.1-8.3 (m, 11H, 2Ph, H-5, and H-7-8), } 9.1(\text { broad, s, } 1 \mathrm{H}, \mathrm{NH}), 2.3(\mathrm{~d}, 1 \mathrm{H}, \mathrm{CH}=\mathrm{CH}), 2.6(\mathrm{~d}, \\
1 \mathrm{H}, \mathrm{CH}=\mathrm{CH}), 10.1(\mathrm{~s}, 1 \mathrm{H}, \mathrm{Ar}-\mathrm{OH}), 2.4(1 \mathrm{H}, \mathrm{d}, \mathrm{N}-\mathrm{CH}), 4.4(1 \mathrm{H}, \mathrm{d}, \mathrm{CH}-\mathrm{Cl})\end{array}$ \\
\hline $5 e$ & $\begin{array}{l}\text { 7.2-8.2(m, } 11 \mathrm{H}, 2 \mathrm{Ph}, \mathrm{H}-5 \text {, and H-7-8), } 9.1(\text { broad, s, } 1 \mathrm{H}, \mathrm{NH}), 2.4(\mathrm{~d}, 1 \mathrm{H}, \mathrm{CH}=\mathrm{CH}), 2.7(\mathrm{~d}, \\
1 \mathrm{H}, \mathrm{CH}=\mathrm{CH}), 10.2(\mathrm{~s}, 1 \mathrm{H}, \mathrm{Ar}-\mathrm{OH}), 2.5(1 \mathrm{H}, \mathrm{d}, \mathrm{N}-\mathrm{CH}), 4.6(1 \mathrm{H}, \mathrm{d}, \mathrm{CH}-\mathrm{Cl})\end{array}$ \\
\hline $5 f$ & $\begin{array}{l}\text { 7.0-8.2 (m, 11H, 2Ph, H-5, and H-7-8), 9.2 (broad, s, } 1 \mathrm{H}, \mathrm{NH}), 2.5(\mathrm{~d}, 1 \mathrm{H}, \mathrm{CH}=\mathrm{CH}), 2.8(\mathrm{~d}, \\
1 \mathrm{H}, \mathrm{CH}=\mathrm{CH}), 2.6(1 \mathrm{H}, \mathrm{d}, \mathrm{N}-\mathrm{CH}), 4.4(1 \mathrm{H}, \mathrm{d}, \mathrm{CH}-\mathrm{Cl})\end{array}$ \\
\hline $5 \mathrm{~g}$ & $\begin{array}{l}\text { 7.0-8.1 (m, 11H, 2Ph, H-5, and H-7-8), } 9.1(\text { broad, } \mathrm{s}, 1 \mathrm{H}, \mathrm{NH}), 2.4(\mathrm{~d}, 1 \mathrm{H}, \mathrm{CH}=\mathrm{CH}), 2.7(\mathrm{~d}, \\
1 \mathrm{H}, \mathrm{CH}=\mathrm{CH}), 2.4(1 \mathrm{H}, \mathrm{d}, \mathrm{N}-\mathrm{CH}), 4.5(1 \mathrm{H}, \mathrm{d}, \mathrm{CH}-\mathrm{Cl})\end{array}$ \\
\hline $5 \mathbf{h}$ & $\begin{array}{l}\text { 7.2-8.3 (m, } 11 \mathrm{H}, 2 \mathrm{Ph}, \mathrm{H}-5 \text {, and H-7-8), } 9.1 \text { (broad, s, } 1 \mathrm{H}, \mathrm{NH}), 2.3(\mathrm{~d}, 1 \mathrm{H}, \mathrm{CH}=\mathrm{CH}), 2.8(\mathrm{~d} \text {, } \\
1 \mathrm{H}, \mathrm{CH}=\mathrm{CH}), 2.5(1 \mathrm{H}, \mathrm{d}, \mathrm{N}-\mathrm{CH}), 4.5(1 \mathrm{H}, \mathrm{d}, \mathrm{CH}-\mathrm{Cl})\end{array}$ \\
\hline $5 i$ & $\begin{array}{l}\text { 7.1-8.4 (m, 11H, 2Ph, H-5, and H-7-8), } 9.2(\text { broad, s, } 1 \mathrm{H}, \mathrm{NH}), 2.4(\mathrm{~d}, 1 \mathrm{H}, \mathrm{CH}=\mathrm{CH}), 2.9(\mathrm{~d}, \\
1 \mathrm{H}, \mathrm{CH}=\mathrm{CH}), 3.8\left(\mathrm{~s}, 1 \mathrm{H}, \mathrm{Ar}-\mathrm{OCH}_{3}\right), 2.4(1 \mathrm{H}, \mathrm{d}, \mathrm{N}-\mathrm{CH}), 4.4(1 \mathrm{H}, \mathrm{d}, \mathrm{CH}-\mathrm{Cl})\end{array}$ \\
\hline $5 \mathbf{j}$ & $\begin{array}{l}7.0-8.3(\mathrm{~m}, 11 \mathrm{H}, 2 \mathrm{Ph}, \mathrm{H}-5 \text {, and H-7-8), } 9.1(\text { broad, s, } 1 \mathrm{H}, \mathrm{NH}), 2.3(\mathrm{~d}, 1 \mathrm{H}, \mathrm{CH}=\mathrm{CH}), 2.8(\mathrm{~d}, \\
1 \mathrm{H}, \mathrm{CH}=\mathrm{CH}), 3.7\left(\mathrm{~s}, 1 \mathrm{H}, \mathrm{Ar}-\mathrm{OCH}_{3}\right), 2.6(1 \mathrm{H}, \mathrm{d}, \mathrm{N}-\mathrm{CH}), 4.4(1 \mathrm{H}, \mathrm{d}, \mathrm{CH}-\mathrm{Cl})\end{array}$ \\
\hline 5 & $\begin{array}{l}\text { 7.2-8.2(m, } 11 \mathrm{H}, 2 \mathrm{Ph}, \mathrm{H}-5 \text {, and H-7-8), } 9.2(\text { broad, } \mathrm{s}, 1 \mathrm{H}, \mathrm{NH}), 2.4(\mathrm{~d}, 1 \mathrm{H}, \mathrm{CH}=\mathrm{CH}), 2.6(\mathrm{~d} \text {, } \\
1 \mathrm{H}, \mathrm{CH}=\mathrm{CH}), 2.4(1 \mathrm{H}, \mathrm{d}, \mathrm{N}-\mathrm{CH}), 4.5(1 \mathrm{H}, \mathrm{d}, \mathrm{CH}-\mathrm{Cl})\end{array}$ \\
\hline 51 & $\begin{array}{l}\text { 7.0-8.2 (m, 16H, 3Ph, H-5, and H-7-8), } 9.1 \text { (broad, s, } 1 \mathrm{H}, \mathrm{NH}), 2.5(\mathrm{~d}, 1 \mathrm{H}, \mathrm{CH}=\mathrm{CH}), 2.8(\mathrm{~d}, \\
1 \mathrm{H}, \mathrm{CH}=\mathrm{CH}), 2.6(1 \mathrm{H}, \mathrm{d}, \mathrm{N}-\mathrm{CH}), 4.6(1 \mathrm{H}, \mathrm{d}, \mathrm{CH}-\mathrm{Cl})\end{array}$ \\
\hline
\end{tabular}


Preparation of 2-[2-\{(4-substitutedbenzylidene)-phenyl\} vinyl]-4-oxo-3, 4-dihydroquinazolone-6sulfonic acid $5(a-l)$

In a $250 \mathrm{~mL}$ R.B.F 2-[2-\{(4-substitutedbenzylidene)-phenyl $\}$ vinyl]-4-oxo-3, 4-dihydroquinazolone-6-sulfonic acid 4(a-l) $(0.001 \mathrm{M})$ and Triethylamine $(0.296 \mathrm{~g}, 0.003 \mathrm{M})$ were dissolved in DMF, Chloroacetyl Chloride $(0.097 \mathrm{~g}$, $0.0012 \mathrm{M})$ in DMF was added dropwise at $0-5^{\circ} \mathrm{C}$. The reaction mixture was stirred for $2 \mathrm{hrs}$ and refluxed for $10-12 \mathrm{hrs}$. Half the solvent was then removed by distillation and cooled. The solid separated was recrystallised from glacial acetic acid, $(60 \%)$. The \% yield is 75\%. IR: $v$ max $1630(\mathrm{C}=\mathrm{N}), 1710(\mathrm{C}=\mathrm{O}), 1420 \quad(-\mathrm{CH}=\mathrm{CH}-), 1730(\mathrm{C}=\mathrm{O}), 785(\mathrm{C}-\mathrm{Cl})$; ${ }^{1} \mathrm{H}$ NMR (CDCl3+DMSO; TMS as internal standard): $\delta 7.1-8.1$ (m, 11H, 2Ph, H-5, and H-7-8), $\delta 9.1$ (broad, s, $1 \mathrm{H}$, $\mathrm{NH}), \delta 2.5(\mathrm{~d}, 1 \mathrm{H}, \mathrm{CH}=\mathrm{CH}), \delta 2.7(\mathrm{~d}, 1 \mathrm{H}, \mathrm{CH}=\mathrm{CH}), \delta 2.5(1 \mathrm{H}, \mathrm{d}, \mathrm{N}-\mathrm{CH}), \delta 4.45(1 \mathrm{H}, \mathrm{d}, \mathrm{CH}-\mathrm{Cl})$.

\section{Result and Discussion}

Antibacterial and antifungal activity

All the compounds $(\mathbf{4 a - 4 l} \& \mathbf{5 a - 5 1 )}$ from the series were screened for their antibacterial activity against B. subtillis, S. aureus, P. aeruginosa and E. coli. The standard drug used was ampicillin, amoxicillin and penicillin and DMF was kept as solvent control. Similarly the antifungal studies were carried out against fungus C. albicans and C. krusei, using Flucanozole as standard. Compounds 4e, 4h, 4i, 4j, 5a, 5f, 5h and 5k showed significant antibacterial activity. Compound $4 \mathrm{~d}, \mathbf{4 e}, \mathbf{4 f}, \mathbf{4 l}, \mathbf{5 a}, \mathbf{5 e}, \mathbf{5 f}$ and $5 \mathrm{l}$ showed significant antifungal activity. The antibacterial and antifungal activity of compounds $\mathbf{4}(\mathbf{a}-\mathbf{l})$ and $\mathbf{5}(\mathbf{a}-\mathbf{l})$ are given in Table-III.

Table-III: - Antibacterial and Antifungal Activity of compound 4(a-1) \& 5(a-l)

\begin{tabular}{|c|c|c|c|c|c|c|}
\hline \multirow{3}{*}{ compound } & \multicolumn{4}{|c|}{ Antibacterial in $(\mu \mathrm{g} / \mathrm{ml})$} & \multicolumn{2}{|c|}{ Antifungal in $(\mu \mathrm{g} / \mathrm{ml})$} \\
\hline & \multicolumn{2}{|c|}{ Gram positive } & \multicolumn{2}{|c|}{ Gram negative } & \multirow{2}{*}{$\begin{array}{c}\text { Candida } \\
\text { ATCC } 10231\end{array}$} & \multirow{2}{*}{$\begin{array}{c}\text { C.krusei } \\
\text { G03 }\end{array}$} \\
\hline & S.a & B.s & E.c & P.a & & \\
\hline $4 a$ & 7 & 12 & 19 & 13 & - & 15 \\
\hline $4 \mathrm{~b}$ & 9 & 0.8 & 6 & 0.9 & 10 & 0.9 \\
\hline $4 c$ & 15 & - & 12 & 9 & 15 & 9 \\
\hline $4 d$ & 8 & 7 & 10 & - & 22 & - \\
\hline $4 \mathrm{e}$ & - & 20 & 9 & 28 & - & 21 \\
\hline $4 \mathrm{f}$ & 15 & 0.9 & 8 & - & 0.9 & 0.4 \\
\hline $4 \mathrm{~g}$ & 8 & - & 0.2 & - & 10 & 25 \\
\hline $4 \mathrm{~h}$ & 28 & 7 & 12 & 10 & - & 8 \\
\hline $4 i$ & - & 25 & - & 21 & 0.7 & 7 \\
\hline $4 j$ & 8 & 12 & 28 & 8 & 7 & 0.8 \\
\hline $4 \mathrm{k}$ & 9 & - & 10 & - & - & 8 \\
\hline 41 & 12 & 15 & 0.8 & 12 & 21 & 10 \\
\hline $5 \mathrm{a}$ & 8 & 10 & 21 & 15 & - & 20 \\
\hline $5 b$ & 7 & 0.8 & 8 & 1.1 & 12 & 0.8 \\
\hline $5 c$ & 12 & - & 15 & 10 & 17 & 9 \\
\hline $5 \mathrm{~d}$ & 7 & 9 & 12 & 12 & - & 12 \\
\hline $5 \mathrm{e}$ & 5 & 15 & 0.9 & 1.8 & 20 & - \\
\hline $5 f$ & 10 & 20 & - & 18 & 0.9 & 20 \\
\hline $5 \mathrm{~g}$ & 12 & 1.1 & 0.9 & - & 10 & 17 \\
\hline $5 \mathrm{~h}$ & 15 & 20 & 12 & 12 & 17 & 8 \\
\hline $5 \mathrm{i}$ & - & 15 & - & 17 & 10 & 0.7 \\
\hline $5 \mathrm{j}$ & 9 & 10 & 12 & 9 & 7 & 0.8 \\
\hline $5 \mathrm{k}$ & 28 & - & 10 & - & 10 & - \\
\hline 51 & 12 & 10 & 0.9 & 10 & 17 & 18 \\
\hline \multicolumn{7}{|c|}{ Zone of inhibition of standard drugs $(\mu \mathrm{g} / \mathrm{ml})$} \\
\hline Ampicillin & 40 & 45 & 40 & 50 & - & - \\
\hline Amoxicillin & 35 & 40 & 38 & 45 & - & - \\
\hline Penicillin & 40 & 38 & 42 & 48 & - & - \\
\hline Flucanozole & - & - & - & - & 40 & 35 \\
\hline
\end{tabular}

S.a- S.aureus, B.s- B.subtilis, E.c-E.coli, P.a-P.aeruginosa 

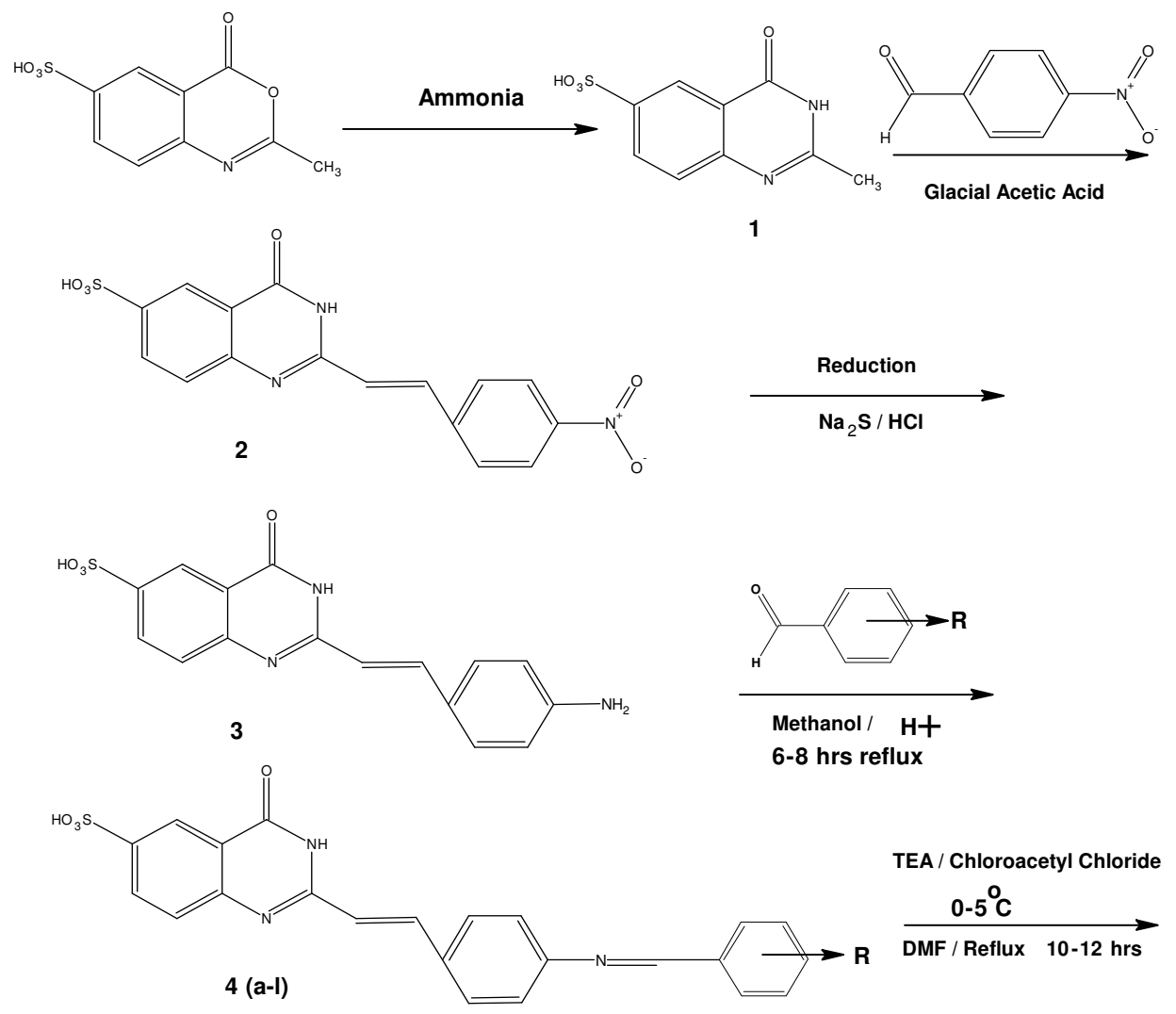

TEA / Chloroacetyl Chloride

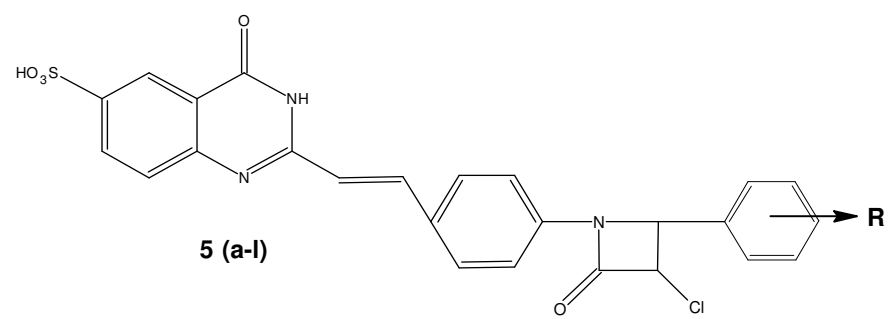

Where $\mathrm{R}=4-\mathrm{N}\left(\mathrm{CH}_{3}\right)_{2} ; 4-\mathrm{Cl}$; 2-Cl; 4-OH; 2-OH; 4- $\mathrm{NO}_{2} ; 2-\mathrm{NO}_{2} ; 2-\mathrm{Br} ; 3-\mathrm{OH}$, $4-\mathrm{OCH}_{3} ; 3-\mathrm{OCH}_{3} ; 2: 4-\mathrm{Cl}_{2} ; 3-\mathrm{OC}_{6} \mathrm{H}_{5}$

\section{Conclusion}

All compounds were screened for their antifungal against Candida albicans and antibacterial against S.aureus, B.subtilis, E.coli and P.aeruginosa. Zone of inhibition were measured in millimeters. The antifungal activities of the test compounds were compared to the standard drug flucanozole (3540mm). DMF was used as solvent. All compounds have shown good activity against candida albicans. Ampicillin (40-50mm), Amoxicillin (35-45mm) and penicillin (38-48mm) were used as standard drugs for antibacterial activity. Compounds $4 \mathbf{e}, \mathbf{4 h}, \mathbf{4 i}, \mathbf{4 j}, \mathbf{5 a}, \mathbf{5 f}, \mathbf{5 h}$ and $\mathbf{5 k}$ showed significant antibacterial activity. Compound 4d, 4e, 4f, 4l, 5a, 5e, 5 f and $5 \mathbf{l}$ showed significant antifungal activity (Table-III). 


\section{Acknowledgment}

The authors are thankful to the Head, Department of chemistry, Veer Narmad South Gujarat University, Surat for providing research facilities and Screening of IR Spectra. The authors are also thankful to Quest institute of life sciences, C/O Nicholas Piramal ltd, Mumbai for screening of antifungal and antibacterial activity and CDRI, Lucknow for providing ${ }^{1} \mathrm{H}$ NMR spectra and elemental analysis.

\section{References}

1. Satsangi R K, Indian Drugs 1979, 17, 79-81.

2. Joshi V and Chaudhari R P, Indian J Chem 1987, 26B, 602-604.

3. Srivastava V K, Gulati S S and Shanker K, Indian J Chem 1987, 26B, 652-656.

4. Gupta D P, Ahmad S, Kumar A and Shanker K, Indian J Chem 1988, 27B, 1060-1062.

5. Sakai K, Nahata H, Jpn Kokai Tokyo Koho J P, 6351, 329, Chem Abstr 1988, 109, 86338.

6. Niementowski V J, Prakt Chem 1895, 51, 564; Beilstein, 24, 143.

7. Pawar R A and Patil A A, Indian J Chem 1994, 33B, 156.

8. Dincer S, Indian J Chem 1996, 35B, 1335.

9. Saxena R K and Khan M A, Indian J Chem 1989, 26B, 443.

10. Gujral M L, Saxena P N and Kohil R P, Indian J Med Res 1957, 215, 207.

11. Surrey A R and Culter R A, J Am Chem Soc 1958, 80, 578.

12. Saxena R K and Pandam K, J Indian Chem Soc 1984, LXI, 722. 


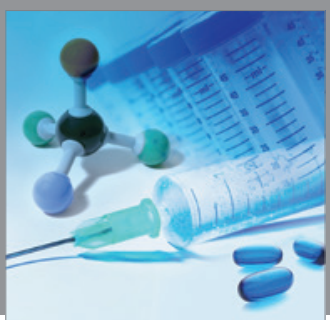

International Journal of

Medicinal Chemistry

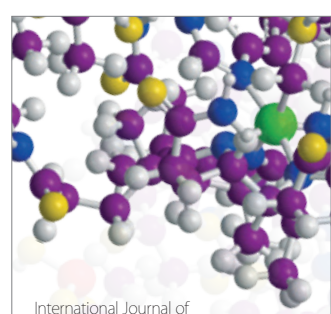

Carbohydrate Chemistry

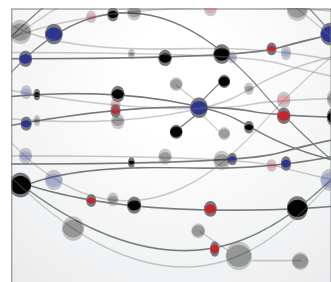

The Scientific World Journal
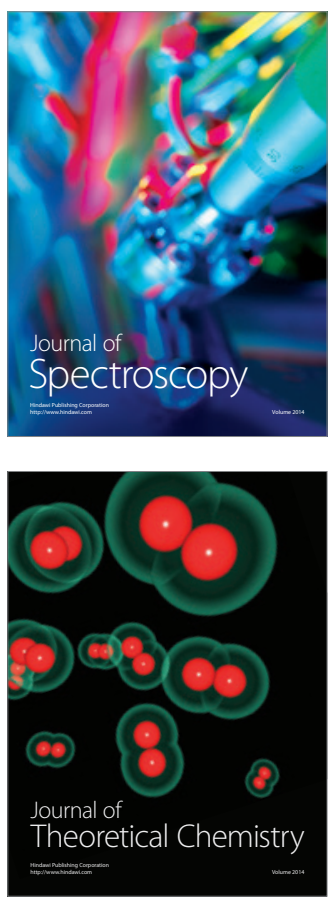
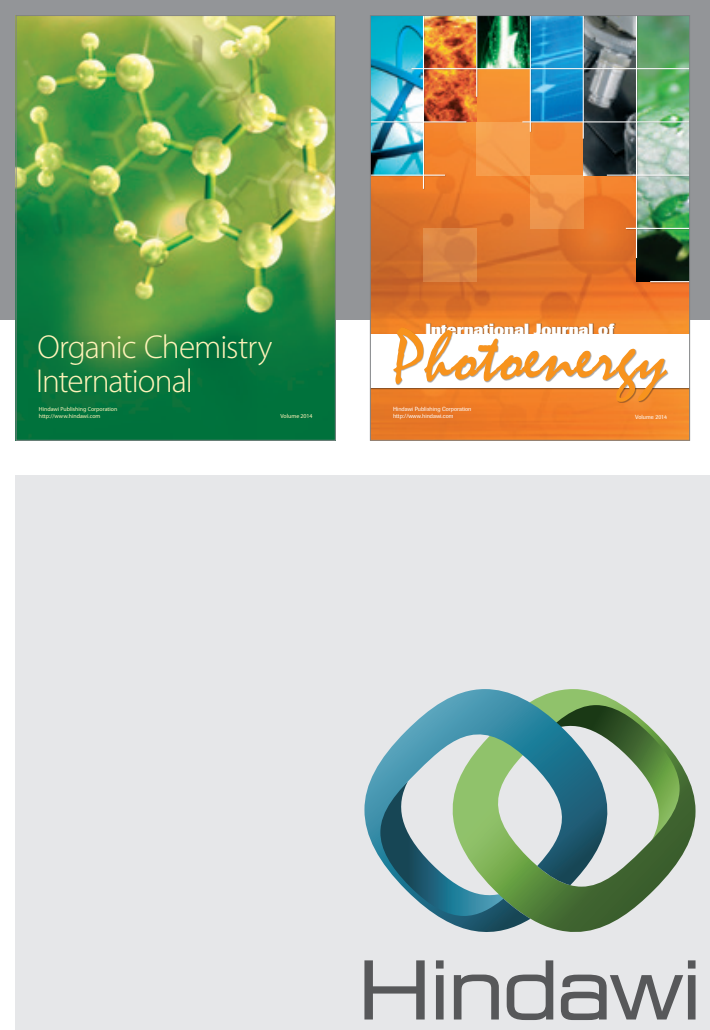

Submit your manuscripts at

http://www.hindawi.com
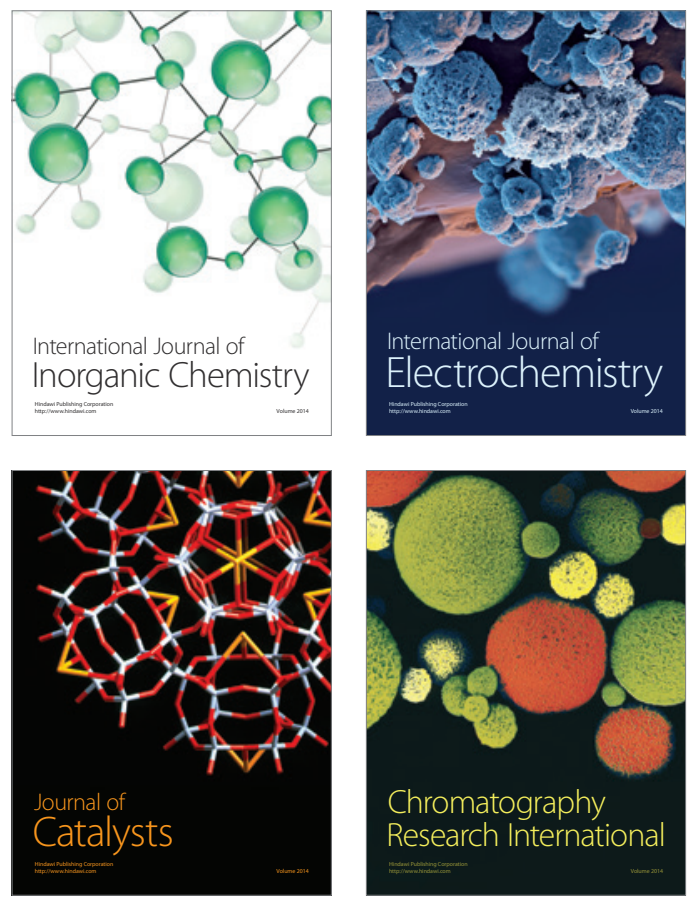
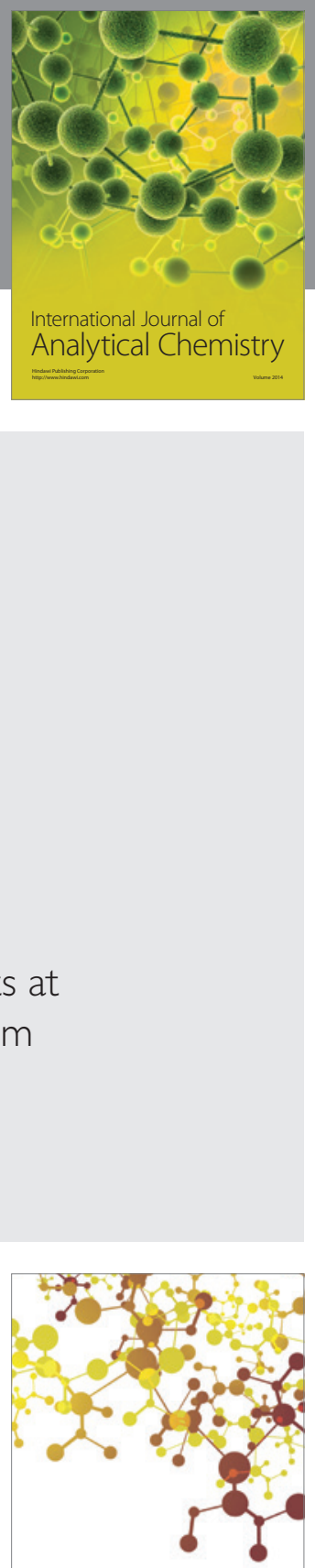

Journal of

Applied Chemistry
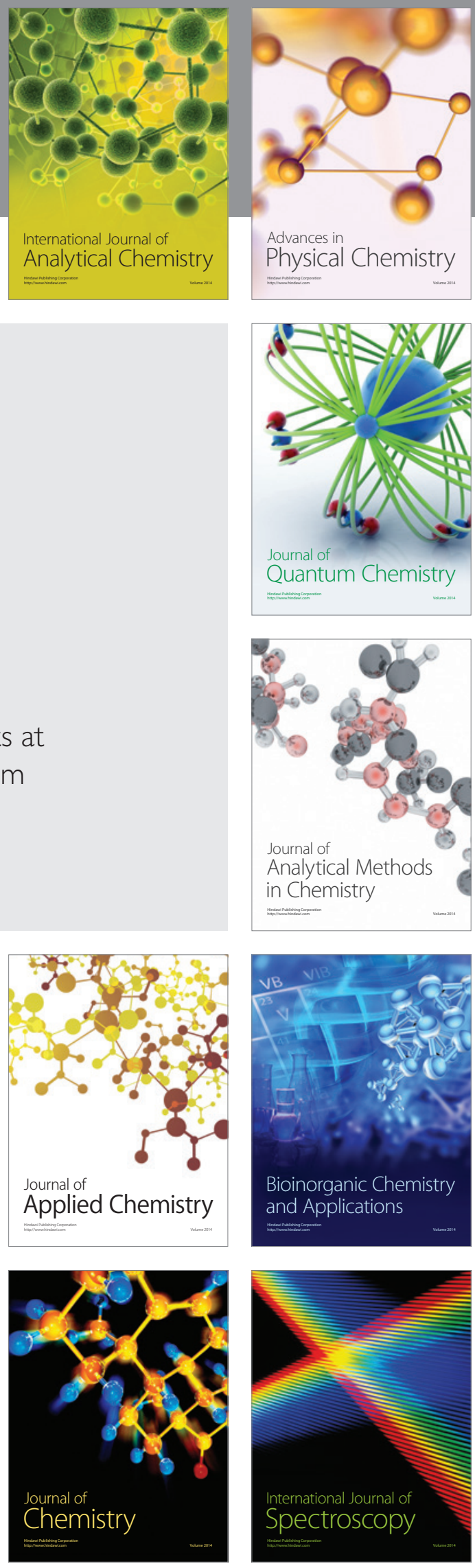